\title{
ODPM Channel Estimation Method using Multiple MRC and New Reliability Test in IEEE 802.11p Systems with Receive Diversity
}

\author{
Sungmook Lim ${ }^{1}$, Gihoon $\mathrm{Ryu}^{2}$, and Kyunbyoung $\mathrm{Ko}^{\mathbf{1}^{*}}$ \\ ${ }^{1}$ Department of IT-Energy Convergence (BK21 FOUR) and Department of Electronics Engineering, \\ Korea National University of Transportation, 50 Daehak-ro, Chungju-si, Chungbuk, 27469 Korea \\ [e-mail: \{smlim, kbko\}@ut.ac.kr] \\ 2 Department of IT-Energy Convergence (BK21 FOUR), Korea National University of Transportation, \\ 50 Daehak-ro, Chungju-si, Chungbuk, 27469 Korea \\ [e-mail: ryugh24@ut.ac.kr] \\ ${ }^{*}$ Corresponding author: Kyunbyoung Ko
}

Received August 10, 2021; revised October 19, 2021; accepted November 22, 2021; published December 31, 2021

\begin{abstract}
In IEEE 802.11p-based wireless access in vehicular environments (WAVE) communication systems, channel estimation (CE) is one of the important issues to provide stable communication service. It is hard to apply conventional CE schemes based on data pilot to real systems, because error propagation occurs in high mobility and modulation order environments, resulting in degrading the CE performance. In this paper, we propose one data pilot using multiple receive antennas (ODPM) CE scheme based on the weighted sum using update matrix (WSUM) with time-domain averaging (TDA) to overcome this problem. Within the process of WSUM-TDA in the proposed scheme, the maximum ratio combining (MRC) technique is applied so as to create more accurate one data pilot. Moreover, a new reliability test criterion is proposed as the fashion of utilizing MRC, which makes it possible to apply selective TDA that guarantees performance improvement. In simulation results, the packet error rate (PER) performance of the proposed ODPM is compared with that of conventional CE methods and its superiority is demonstrated.
\end{abstract}

Keywords: IEEE 802.11p, V2X, ODPM, WAVE-TDA, MRC

A preliminary version of this paper appeared in 2018 International Conference on Convergence Content (ICCC2018), Jeju, Korea, 17-19, Dec., 2018. This version includes a concrete analysis and an improved algorithm of the channel estimation. 


\section{Introduction}

Recently, interest in vehicle-to-everything (V2X) communication systems has explosively increased because of providing reliable traffic safety and infotainment service in the highspeed moving environment of vehicles [1-17].

In order to reliably provide V2X, the standardization of wireless access in vehicular environments (WAVE) based on IEEE 802.11p has proceeded, where physical and medium access control layers are defined [1][2]. It is noted that the IEEE 802.11p is the same as the IEEE802.11a, which was standardized for a static indoor environment, except that the bandwidth is reduced from $20 \mathrm{MHz}$ to $10 \mathrm{MHz}$ [1]. In other words, the IEEE 802.11p has only long training symbols in preamble of each frame for channel estimation (CE) and only four phase tracking pilot subcarriers, which are used for compensating phase rotation, in each orthogonal frequency division multiplexing (OFDM) symbol. It causes inaccurate CE by the channel's time-varying characteristics in the high-speed moving environment of vehicles, resulting in degrading the packet error rate (PER) performance. In order to overcome this problem, various data pilot-based CE schemes in IEEE 802.11p-based WAVE systems are being studied, where the estimated channel values in frequency domain are updated based on the estimated data symbols for every OFDM symbol. [3-14].

In single receive antenna environment, one of representative data pilot-based CE schemes is the spectral temporal averaging (STA) scheme, where the estimated channel coefficient is averaged in both the frequency and the time domains [4]. Furthermore, in [4], the construct data pilot (CDP) scheme was proposed in order to enhance the PER performance in high signal to noise ratio (SNR) by performing the reliability test. In [5], time-domain reliable-test frequency-domain interpolation (TRFI) was proposed in order to alleviate the error propagation problem of CDP by improving the reliability test. On the other hand, the weighted sum (WSUM) scheme was also proposed to enhance the PER performance in low SNR by introducing a novel update matrix [8], and the CE scheme based on minimum mean square error (MMSE) was proposed, which can be implemented in a real environment [9]. However, in all of the existing methods mentioned above, the PER performance can be degraded in higher modulation order and time-selectivity of the channel.

On the other hand, in multiple receive antenna environment, the estimated channel coefficients are updated by maximal ratio combining (MRC) based on data pilot constructed by each antenna, resulting in enhancing PER due to receive diversity gain [10-14]. In [10] and [11], the authors studied the receiver and transmit diversities in IEEE 802.11p standard and MRC technique was applied based on independent CE for each receiving antenna. In [12], the authors proposed the receiver structure using a decision feedback equalizer (DFE) based on decoded bits and MRC so that it required both high processing delay and complexity. In [14], the authors proposed the iterative CE scheme based on MRC packing CE scheme but it does not fully obtain MRC diversity in CE process.

As far as we know, there have been no studies on CE methods in multiple receive antenna environment where receive antenna diversity can be sufficiently obtained during the $\mathrm{CE}$ process to provide robustness in all SNR regions, regardless of modulation schemes.

In this paper, we propose one data pilot using multiple receive antennas (ODPM) CE scheme based on WSUM with time-domain averaging (WSUM-TDA) and then, the performance enhancement is evaluated. Also, we compare the performance of conventional MRC based CE schemes. An important feature of the proposed scheme is that MRC technique is applied in three part. In the first part, WSUM-TDA scheme is applied so that it has a structure that makes one constructed data pilot by applying MRC instead of independently making 
constructed data pilots for each receive antenna. The second part is related to a new reliability test using two variables for each antenna and two variables applying MRC. Finally, MRC is applied to the received signals using the estimated channel value per antenna.

The remainder of this paper is organized as follows: In Section 2, physical layer of the IEEE 802.11p standard is described. Then, Section 3 addresses conventional CE schemes. Section 4 proposes a new CE scheme. In Section 5, simulation results are shown. Finally, in Section 6, concluding remarks are given.

\section{System Model}

The IEEE 802.11p standard is basically designed the same as IEEE 802.11a [1]. It is based on OFDM at the center frequency of $5.9 \mathrm{GHz}$. When fast Fourier transform (FFT) and inverse FFT is performed, its size is 64 . In the transmitter, the convolutional encoder with constraint length 7 is used as a channel coding, and the Viterbi decoder is used in the receiver. The main difference between the IEEE 802.11p and the IEEE 802.11a is that it uses a bandwidth of $10 \mathrm{MHz}$ whereas the IEEE 802.11a utilizes 20MHz. Because of that, the IEEE 802.11p utilizes the subcarrier frequency spacing of $0.15625 \mathrm{MHz}$, whereas the IEEE 802.11a utilizes $0.3125 \mathrm{MHz}$. In addition, the FFT period, guard interval (GI) duration and symbol duration are twice the size of them of the IEEE 802.11a [1].

In the IEEE 802.11p, the frame structure consists of three parts, which is also the same as the IEEE 802.11a: 1) preamble which is divided into short and long training symbols for time synchronization and CE, respectively, 2) signal field which includes control information such as the modulation order, the channel code rate and so on, and 3) data field which consists of multiple OFDM symbols which conveys the transmitted information. The number of OFDM symbols constituting data filed is variable according to systems. [1][9].

Each OFDM symbol is composed of 64 subcarriers of which the frequency index ranges from -32 to 31 . The 64 subcarriers of each OFDM consist of 4 as pilot subcarriers, 12 as null subcarriers and 48 as data subcarriers, respectively. The original purpose of 4 pilot subcarriers is to compensate the phase rotation. However, in the data pilot-based CE schemes, they are also used for estimating the channel coefficients by tracking the time variation of the channel in frequency domain. They are located on -21, -7, 7 and 21 in the frequency index. The 12 null subcarriers consist of 11 virtual subcarriers where the corresponding subcarrier indices are from -32 to -27 and from 28 to 31, and a direct current subcarrier which is located on subcarrier 0 . The remaining 48 data subcarriers are used for data transmission. In this paper, the subcarrier indices of -21, -7, 7 and 21 are defined as $S_{p}$ and the set of data subcarrier indices is defined as $S_{d}$, which satisfies $S_{d} \cup S_{p}=\{-26,-25, \cdots,-1,1, \cdots, 25,26\}$. For other specific parameters defined in [1].

In the receiver, after removing GI and performing FFT, the signal at the $k$ th subcarrier of the $i$ th OFDM symbol in $r$ th rx antenna, $Y_{r, i}(k)$, is

$$
Y_{r, i}(k)=H_{r, i}(k) X_{i}(k)+W_{r, i}(k)
$$

with $r \in\{1,2, \cdots, R\}, i \in\left\{1,2, \cdots, N_{\text {data }}\right\}$, and $k \in S_{d} \cup S_{p} . R$ and $N_{\text {data }}$ are the numbers of receive antenna and OFDM symbols in data field (i.e., packet), respectively. $H_{r, i}(k), X_{i}(k)$, and $W_{r, i}(k)$ mean the channel coefficient, the data symbol, and the additive white Gaussian noise (AWGN) with zero mean and variance $\sigma_{W}^{2}$ in the frequency domain, respectively. 


\section{Conventional CE Schemes for the IEEE 802.11p}

In this section, the conventional CE techniques including STA, CDP and WSUM are explained and analyzed. [4][8][9].

\subsection{STA}

In STA, the estimated channel coefficients are averaged on both time and frequency axes to overcome the performance degradation due to time-varying channels. [4].

\subsubsection{Equalization}

At first, for the $r$ th rx antenna, the channel coefficient of the $k$ th subcarrier, $\hat{H}_{r, 0}(k)$, is estimated by the least square (LS) method using two received long training symbols in preamble, which are denoted as $Y_{r, 1}^{T}(k)$ and $Y_{r, 2}^{T}(k)$. Then, $\hat{H}_{r, 0}(k)$ can be expressed as follows:

$$
\hat{H}_{r, 0}(k)=\frac{Y_{r, 1}^{T}(k)+Y_{r, 2}^{T}(k)}{2 X^{T}(k)}, k \in\left(S_{d} \cup S_{p}\right)
$$

Here, $X^{T}(k)$ is the long training symbol of the $k$ th subcarrier in the transmitter, which the receiver also knows.

In order to equalize the received signal of the $k$ th subcarrier at the $r$ th antenna in the $i$ th OFDM symbol, $Y_{r, i}(k)$, the previous $(i-1)$ th channel coefficient, $\hat{H}_{r, i-1}^{\text {STA }}(k)$, is used as follows:

$$
S_{r, i}(k)=Y_{r, i}(k) / \hat{H}_{r, i-1}^{\mathrm{STA}}(k), \quad k \in S_{d}
$$

When $i=1, \hat{H}_{r, 0}^{\text {STA }}(k)$ is equal to $\hat{H}_{r, 0}(k)$ in (2). Also, it is noted for $i=1$ that we can set $\hat{H}_{r, 0}^{\mathrm{STA}}(k)=\hat{H}_{r, 0}^{\mathrm{FDA}}(k)$ from (6).

\subsubsection{Construction of Data Pilot}

As a next step, based on $S_{r, i}(k)$ in (3), $\hat{X}_{r, i}(k)$, is demapped as

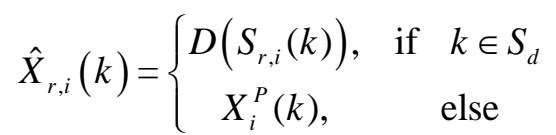

where $\hat{X}_{r, i}(k)$ denotes the data symbol and $D(\cdot)$ means a demapping quantizer which maps the equalized signal to the nearest constellation point. $X_{i}^{P}(k)$ with $k \in S_{p}$ is a predefined pilot symbol in the frequency domain. Then, in the next step, $\hat{X}_{r, i}(k)$ can be used to update the estimated channel coefficient as data pilot.

\subsubsection{LS}

Based on the constructed data pilot, $\hat{X}_{r, i}(k)$ in (4), the channel coefficient of the $k$ th subcarrier at the $r$ th antenna in the $i$ th OFDM symbol, $\hat{H}_{r, i}(k)$, is estimated as follows:

$$
\hat{H}_{r, i}(k)=Y_{r, i}(k) / \hat{X}_{r, i}(k), k \in\left(S_{d} \cup S_{p}\right) \text {. }
$$




\subsubsection{Averaging in Frequency Domain}

The estimated channel coefficient in (5) can be distorted by demapping error as well as AWGN. In order to alleviate this distortion, the adjacent channel coefficients in the frequency axis are averaged as

$$
\hat{H}_{r, i}^{\mathrm{FDA}}(k)=\sum_{\lambda=-\beta}^{\beta} \hat{H}_{r, i}(k+\lambda) \omega_{\lambda}, \quad k \in\left(S_{d} \cup S_{p}\right)
$$

where $\omega_{\lambda}=1 /(2 \beta+1)$ is a weighting coefficient and $(2 \beta+1)$ means the number of subcarriers used for averaging [4][8].

\subsubsection{Averaging in Time Domain}

After averaging the channel coefficients in the frquency domain, we apply time domain averaging for $i>0$. Then, the channel coefficient can be finally estimated as

$$
\hat{H}_{r, i}^{\mathrm{STA}}(k)=\left(1-\frac{1}{\alpha}\right) \hat{H}_{r, i-1}^{\mathrm{FDA}}(k)+\frac{1}{\alpha} \hat{H}_{r, i}^{\mathrm{FDA}}(k)
$$

for $k \in\left(S_{d} \cup S_{p}\right)$ and $\alpha$ is a weight coefficient in the time domain. In this paper, we consider $\alpha=\beta=2$ for STA scheme [4][8].

The finally estimated channel coefficient, $\hat{H}_{r, i}^{\text {STA }}(k)$ in (7) is used to construct data pilot and update the channel coefficient at the next $(i+1)$ th OFDM symbol. Therefore, we return to the procedure of (3) and repeat the remaining steps. After operations from (2) to (7) are independently performed for each antenna, MRC can be finally performed.

\subsection{CDP}

In CDP scheme, it is assumed that channels between two adjacent OFDM symbols are highly correlated, so channel variation is insignificant. Based on this assumption, the reliability test of the estimated channel coefficient is performed, and the channel coefficients which only pass the reliability test are selected [4]. The CE procedure of CDP is equal to that of STA from (2) to (5). Furthermore, $\hat{H}_{r, 0}^{\mathrm{CDP}}(k)=\hat{H}_{r, 0}(k)$ is used for $i=1$.

\subsubsection{Equalization and Demapping}

In order to perform the reliability test in CDP, the initial channel coefficient to be estimated by (5), $\hat{H}_{r, i}(k)$, and the finally estimated channel coefficient of the previous OFDM symbol, $\hat{H}_{r, i-1}^{\mathrm{CDP}}(k)$, are utilized to equalize the $(i-1)$ th received signal (i.e., $Y_{r, i-1}(k)$ for $k \in S_{d}$ ) as follows [4]:

$$
\begin{aligned}
& S_{r, i-1}^{\prime}(k)=Y_{r, i-1}(k) / \hat{H}_{r, i}(k) \\
& S_{r, i-1}^{\prime \prime}(k)=Y_{r, i-1}(k) / \hat{H}_{r, i-1}^{\mathrm{CDP}}(k)
\end{aligned}
$$

Then, based on $S_{r, i-1}^{\prime}(k)$ and $S_{r, i-1}^{\prime \prime}(k), \hat{X}_{r, i-1}^{\prime}(k)$ and $\hat{X}_{r, i-1}^{\prime \prime}(k)$ can be obtained as [4]

$$
\begin{aligned}
& \hat{X}_{r, i-1}^{\prime}(k)=D\left(S_{r, i-1}^{\prime}(k)\right) \\
& \hat{X}_{r, i-1}^{\prime \prime}(k)=D\left(S_{r, i-1}^{\prime \prime}(k)\right)
\end{aligned}
$$

and, for $k \in S_{p}$, we can set $\hat{X}_{r, i-1}^{\prime \prime}(k)=\hat{X}_{r, i-1}^{\prime}(k)=X_{i}^{P}(k)$. 


\subsubsection{Reliability Test}

Under the assumption of the high channel correlation between two adjacent OFDM symbols in time domain, $\hat{H}_{r, i}(k)$ should be similar to $\hat{H}_{r, i-1}^{\mathrm{CDP}}(k)$. Therefore, if the estimated channel, $\hat{H}_{r, i}(k)$, is correct, $\hat{X}_{r, i-1}^{\prime}(k)$ and $\hat{X}_{r, i-1}^{\prime \prime}(k)$ should be the same. Based on that, the final channel coefficient in CDP can be determined as follows:

$$
\hat{H}_{r, i}^{\mathrm{CDP}}(k)=\left\{\begin{array}{cc}
\hat{H}_{r, i}(k) & \text { if } \hat{X}_{r, i-1}^{\prime}(k)=\hat{X}_{r, i-1}^{\prime \prime}(k) \\
\hat{H}_{r, i-1}^{\mathrm{CDP}}(k) & \text { else. }
\end{array}\right.
$$

The channel coefficient estimated by $(10), \hat{H}_{r, i}^{\mathrm{CDP}}(k)$, can be used to construct data pilot and update the channel coefficient at the next $(i+1)$ th OFDM symbol. Therefore, we return to the procedure of (3) and repeat the remaining steps with $\hat{H}_{r, i-1}^{\mathrm{STA}}(k)=\hat{H}_{r, i-1}^{\mathrm{CDP}}(k)$.

\subsection{WSUM}

In WSUM, an update matrix which indicates whether the channel coefficient estimated by CDP is accurate or not is firstly defined, and based on that, the channel coefficient is updated by weighted averaging procedure [8].

\subsubsection{Initial CE}

When $i=1$, we can set $\hat{H}_{r, 0}^{\mathrm{CDP}}(k)=\hat{H}_{r, 0}(k)$ from (2) and $M_{r, 0}(k)=1$ for $k \in\left(S_{d} \cup S_{p}\right)$. The initial channel coefficient, $\hat{H}_{r, 0}^{\text {wsum }}(k)$, can be estimated as follows:

$$
\hat{H}_{r, 0}^{\mathrm{wSUM}}(k)=\frac{\sum_{\lambda=-\beta}^{\beta} \hat{H}_{r, 0}^{\mathrm{CDP}}(k+\lambda) M_{r, 0}(k+\lambda) \omega_{\lambda}}{\sum_{\lambda=-\beta}^{\beta} M_{r, 0}(k+\lambda) \omega_{\lambda}}
$$

with $\beta=1$ and $\left[\omega_{-1}, \omega_{0}, \omega_{1}\right]=[0.5,1.0,0.5][8][9]$.

\subsubsection{WSUM Equalization}

Similarly to (3), the received signal, $Y_{r, i}(k)$, is equalized by the previous $(i-1)$ th channel coefficient, $\hat{H}_{r, i-1}^{\text {wsum }}(k)$, and the equalized signal is

$$
S_{r, i}(k)=Y_{r, i}(k) / \hat{H}_{r, i-1}^{\mathrm{wsum}}(k), k \in S_{d}
$$

Note that for $i=1, \hat{H}_{r, 0}^{\text {wsum }}(k)$ of (11) is used in (12).

\subsubsection{Construction of Data Pilot}

Through (4) and (12), the data symbol, $\hat{X}_{r, i}(k)$, can be obtained. Furthermore, from (5) with $\hat{X}_{r, i}(k), \hat{H}_{r, i}(k)$ can be easily obtained.

\subsubsection{Equalization and Demapping}

By using $\hat{H}_{r, i}(k)$ and $\hat{H}_{r, i-1}^{\text {wsum }}(k)$ in (8) and (9), the previous received data symbol, $Y_{r, i-1}(k)$, 
is equalized and then demapped for $k \in S_{d}$ as

$$
\begin{aligned}
& \hat{X}_{r, i-1}^{\prime}(k)=D\left(Y_{r, i-1}(k) / \hat{H}_{r, i}(k)\right) \\
& \hat{X}_{r, i-1}^{\prime \prime}(k)=D\left(Y_{r, i-1}(k) / \hat{H}_{r, i-1}^{\text {WSUM }}(k)\right) .
\end{aligned}
$$

For $k \in S_{p}$, we can set $\hat{X}_{r, i-1}^{\prime \prime}(k)=\hat{X}_{r, i-1}^{\prime}(k)=X_{i}^{P}(k)$.

\subsubsection{Weighted SUM}

By usinig (13), we define the update matrix of the $k$ th subcarrier at the $r$ th antenna in the $i$ th OFDM symbol, $M_{r, i}(k)$, as follows:

$$
M_{r, i}(k)=\left\{\begin{array}{cc}
1, & \text { if } \hat{X}_{r, i-1}^{\prime}(k)=\hat{X}_{r, i-1}^{\prime \prime}(k) \\
0, & \text { else. }
\end{array}\right.
$$

In WSUM, it is assumed that channels between adjacent subcarriers in frequency domain are highly correlated. Based on that, the final channel coefficient to be estimated by weighted sum is

$$
\hat{H}_{r, i}^{\mathrm{wsUM}}(k)=\left\{\begin{array}{cc}
\frac{\sum_{\lambda=-\beta}^{\beta} \hat{H}_{r, i}^{\mathrm{CDP}}(k+\lambda) M_{r, i}(k+\lambda) \omega_{\lambda}}{\sum_{\lambda=-\beta}^{\beta} M_{r, i}(k+\lambda) \omega_{\lambda}} & \text { if } \sum_{\lambda=-\beta}^{\beta} M_{r, i}(k+\lambda) \geq N \\
\hat{H}_{r, i-1}^{\mathrm{WSUM}}(k) & \text { else }
\end{array}\right.
$$

with $\beta=1$ and $N=2$ [8][9].

The final channel coefficient estimated by $(15), \hat{H}_{r, i}^{\text {wsum }}(k)$, can be used to construct data pilot and update the channel coefficient at the next $(i+1)$ th OFDM symbol. Therefore, we return to the procedure of (12) and repeat the remaining steps.

\section{Proposed CE Method}

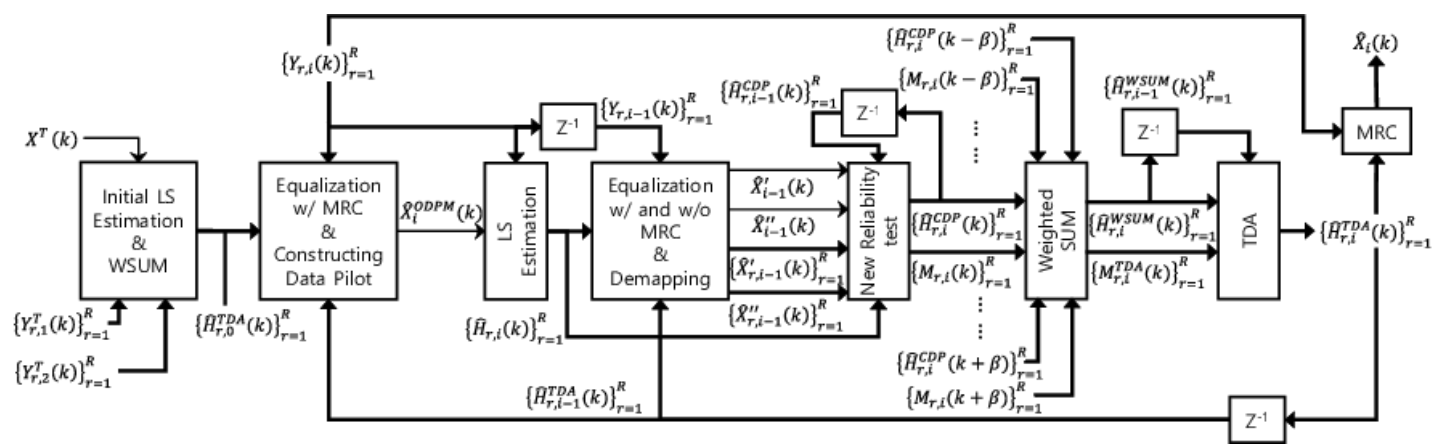

Fig. 1. Block diagram of the proposed CE scheme. 
In the existing studies, the MRC technique is applied only to the step of equalizing the received signal using channel estimates for each receiving antenna. The proposed method utilizes MRC technique in two steps in addition to this. In the first step related with WSUM-TDA scheme, we can obtain one constructed data pilot by applying MRC instead of independently constructed data pilots for each receiving antenna. Note that by making a more accurate constructed data pilot, more accurate $\mathrm{CE}$ can be made in the later process. In second step, we propose a new reliability test criteria to which MRC is applied. Under a new reliability test criteria, we can obtain a more accurate instantaneous CE coefficient, and through this, the CE performance of the WSUM technique is also improved. In addition, the proposed new reliability test criteria is related to the application of selective TDA, which guarantees performance improvement through TDA.

Fig. 1 shows the block diagram of the proposed CE scheme.

\subsection{Initial CE}

It is noted that the proposed CE scheme is based on WSUM-TDA method [9]. For $i=0$, we can get $\hat{H}_{r, 0}^{\mathrm{TDA}}(k)=\hat{H}_{r, 0}^{\mathrm{WSUM}}(k)$ from (2) and (11).

\subsection{ODPM (CDP using MRC)}

For increasing the accuracy of the constructed data pilot, the MRC technique can be applied for $k \in S_{d}$ as

$$
\hat{X}_{i}^{\mathrm{ODPM}}(k)=D\left(\frac{\sum_{r=1}^{R} \hat{H}_{r, i-1}^{\mathrm{TDA}}(k) Y_{r, i}(k)}{\sum_{r=1}^{R}\left|\hat{H}_{r, i-1}^{\mathrm{TDA}}\right|^{2}}\right)
$$

and we can set $\hat{X}_{i}^{\text {ODPM }}(k)=X_{i}^{P}(k)$ for $k \in S_{p}$. By comparing (16) with (4), it is shown that one data pilot symbol is constructed in the proposed scheme [13].

\subsection{Weighted SUM}

\subsubsection{LS Estimation}

From (5) with $\hat{X}_{i}^{\text {ODPM }}(k)$, we can get

$$
\hat{H}_{r, i}(k)=Y_{r, i}(k) / \hat{X}_{i}^{\mathrm{ODPM}}(k), k \in\left(S_{d} \cup S_{p}\right) .
$$

\subsubsection{Equalization and Demapping}

Similar with (13) and (16), for $k \in S_{d}$, we can get

$$
\begin{aligned}
& \hat{X}_{r, i-1}^{\prime}(k)=D\left(Y_{r, i-1}(k) / \hat{H}_{r, i}(k)\right) \\
& \hat{X}_{r, i-1}^{\prime \prime}(k)=D\left(Y_{r, i-1}(k) / \hat{H}_{r, i-1}^{\mathrm{TDA}}(k)\right)
\end{aligned}
$$

and 


$$
\begin{aligned}
& \hat{X}_{i-1}^{\prime}(k)=D\left(\sum_{r=1}^{R} \hat{H}_{r, i}^{*}(k) Y_{r, i-1}(k) / \sum_{r=1}^{R}\left|\hat{H}_{r, i}\right|^{2}\right) \\
& \hat{X}_{i-1}^{\prime \prime}(k)=D\left(\frac{\sum_{r=1}^{R} \hat{H}_{r, i-1}^{\mathrm{TDA} *}(k) Y_{r, i-1}(k)}{\sum_{r=1}^{R}\left|\hat{H}_{r, i-1}^{\mathrm{TDA}}\right|^{2}}\right) .
\end{aligned}
$$

For $k \in S_{p}$, we can set $\hat{X}_{r, i-1}^{\prime \prime}(k)=\hat{X}_{r, i-1}^{\prime}(k)=\hat{X}_{i-1}^{\prime \prime}(k)=\hat{X}_{i-1}^{\prime}(k)=X_{i}^{P}(k)$. Note that, as shown in (19), new variables for the reliability test are obtained regardless of the antenna index by applying MRC in the proposed scheme.

\subsubsection{New Reliability Test}

From (18) and (19), we propose new reliability test criteria and update matrix as

$$
\hat{H}_{r, i}^{\mathrm{CDP}}(k)=\left\{\begin{array}{cc}
\hat{H}_{r, i}(k) & \text { if } \hat{X}_{r, i-1}^{\prime \prime}(k)=\hat{X}_{r, i-1}^{\prime}(k) \text { or } \hat{X}_{i-1}^{\prime \prime}(k)=\hat{X}_{i-1}^{\prime}(k) \\
\hat{H}_{i-1}^{\mathrm{CDP}}(k) & \text { else }
\end{array}\right.
$$

and

$$
M_{r, i}(k)=\left\{\begin{array}{cc}
1, & \text { if } \hat{X}_{r, i-1}^{\prime \prime}(k)=\hat{X}_{r, i-1}^{\prime}(k) \text { or } \hat{X}_{i-1}^{\prime \prime}(k)=\hat{X}_{i-1}^{\prime}(k) \\
0, & \text { else. }
\end{array}\right.
$$

From (15) with both (20) and (21), we can get $\hat{H}_{r, i}^{\text {wsum }}(k)$.

When comparing (20) and (10), we can see the difference in the condition of $\hat{X}_{i-1}^{\prime \prime}(k)=\hat{X}_{i-1}^{\prime}(k)$. When this condition is satisfied in the proposed scheme, it means that $\hat{H}_{r, i}^{\mathrm{CDP}}(k)$ of all antennas are simultaneously updated to instantaneous channel values $\hat{H}_{r, i}(k)$ by fully acquiring the receive antenna diversity. On the other hand, in the conventional method, each antenna can be independently updated, which means that the receive antenna diversity during CDP precess cannot be completely obtained.

\subsection{Selective TDA}

Similar with STA scheme, a TDA method can be also applied into WSUM scheme and it can be expressed as [9]

$$
\hat{H}_{r, i}^{\mathrm{TDA}}(k)=\frac{\hat{H}_{r, i-1}^{\mathrm{WSUM}}(k)+M_{r, i}^{\mathrm{TDA}}(k) \hat{H}_{r, i}^{\mathrm{WSUM}}(k)}{1+M_{r, i}^{\mathrm{TDA}}(k)}
$$

where $M_{r, i}^{\mathrm{TDA}}(k)$ is a variable indicating whether TDA is applied or not and is expressed as

$$
M_{r, i}^{\mathrm{TDA}}(k)=\left\{\begin{array}{cc}
1, & \text { if } \sum_{\lambda=-\beta}^{\beta} M_{r, i}(k+\lambda) \geq N \\
0, & \text { else. }
\end{array}\right.
$$

Finally, from $\left\{H_{r, i}^{\mathrm{TDA}}(k)\right\}_{r=1}^{R}$ of (22), we equalize the received signals by applying MRC as

$$
\hat{X}_{i}(k)=\sum_{r=1}^{R} \hat{H}_{r, i}^{\mathrm{TDA}^{*}}(k) Y_{r, i}(k) / \sum_{r=1}^{R}\left|\hat{H}_{r, i}^{\mathrm{TDA}}\right|^{2} .
$$

Notice in the proposed scheme that, as shown in Fig. 1, MRC technique is used at three 
parts: $\hat{X}_{i}^{\text {ODPM }}(k)$ of (16), both $\hat{X}_{i-1}^{\prime}(k)$ and $\hat{X}_{i-1}^{\prime \prime}(k)$ of (19), and $\hat{X}_{i}(k)$ of (24).

\section{Simulation Results}

In this section, the superiority of the proposed ODPM is demonstrated. For this, V2X channel models presented by Cohda Wireless are considered, where the five scenarios are presented according to the driving environment and the moving speed of vehicles [15]. Among the five scenarios, 'Street Crossing NLOS with $126 \mathrm{~km} / \mathrm{h}$ ', 'Highway LOS with $252 \mathrm{~km} / \mathrm{h}$ ', and 'Highway NLOS with $252 \mathrm{~km} / \mathrm{h}$ ' were used for our simulations. Parameters of each scenario including the channel power, delay and Doppler are refered in [15]. In addition, we adopt QPSK and 16-QAM with coding rate of $1 / 2$. In order to evaluate the PER performance, $10^{5}$ packets are generated and transmitted with $R \in\{2,3\}, N_{\text {data }}=100$, and $\mathrm{SNR}=E\left[\left|H_{r, i}(k)\right|^{2}\right] / \sigma_{W}^{2}$.

In Fig. 2 and Fig. 3, the PER performance of ODPM is compared with that of conventional CE methods under 'Street Crossing NLOS with $126 \mathrm{~km} / \mathrm{h}$ ', when QPSK and 16QAM are considered, respectively. In Fig. 4 and Fig. 5, when 'Highway LOS with $252 \mathrm{~km} / \mathrm{h}$ ' is considered, the PER performance of ODPM is compared with that of conventional CE methods with QPSK and 16QAM modulations, respectively. Finally, in Fig. 6 and Fig. 7, ODPM with QPSK and 16QAM modulations is evaluted in terms of the PER performance in the channel model of 'Highway NLOS with $252 \mathrm{~km} / \mathrm{h}$ '.

In all figures, 'Proposed ODPM' means the proposed scheme in Section 4, where $\beta=1$, $\left[\omega_{-1}, \omega_{0}, \omega_{1}\right]=[0.5,1.0,0.5]$, and $N=2$ [8]. We show the PER performance for conventional existing schemes of 'STA', 'CDP', 'WSUM', and 'WSUM-TDA'. In the existing schemes, the $\mathrm{CE}$ process for each antenna is independently performed and finally the MRC technique is used only once as the same fashion of (24).

Through all the figures, the proposed ODPM CE scheme outperforms all conventional schemes in all SNR regions with regard to the PER performance. Moreover, its superiority has been demonstrated, with both QPSK and 16QAM modulations.

In figures 2, 3, 5 and 6, the PER performances of CDP, WSUM and WSUM-TDA are better than that of STA only when SNR is high. However, ODPM shows the better performance than STA in all SNR regions. Therefore, it is demonstrated that ODPM is resistant to time variation of the channel as well as noise in low SNR region. From Fig. 4 and Fig. 5, it is shown at PER= $10^{-2}$ that the proposed scheme can give more than 2.5dB SNR gain over 'WSUM-TDA' and more than 5.0dB SNR gain over 'WSUM', respectively. Furthermore, it is verified that the performance gain of ODPM will be more pronounced at higher modulation order. Furthermore, from Fig. 6 and Fig. 7, it is shown that the proposed method in the NLOS environment can achieve PER $=10^{-2}$ in a reasonable SNR area.

In Fig. 8 and Fig. 9, the PER performance of the proposed ODPM is compared with that of WSUM-TDA as the number of antennas increases under 'Highway NLOS with $252 \mathrm{~km} / \mathrm{h}$ ' with QPSK and 16QAM modulations, respectively. The more the number of antennas is, the more pronounced the performance enhancement of the proposed scheme due to the diversity gain is. Furthermore, when the number of antennas is 3, the proposed method meets $1 \%$ PER at SNR $=10 \mathrm{~dB}$ and SNR $=18 \mathrm{~dB}$ with QPSK and 16QAM modulations, respectively, whereas the conventional method does not satisfy $1 \%$ PER even at SNR $=30 \mathrm{~dB}$. 


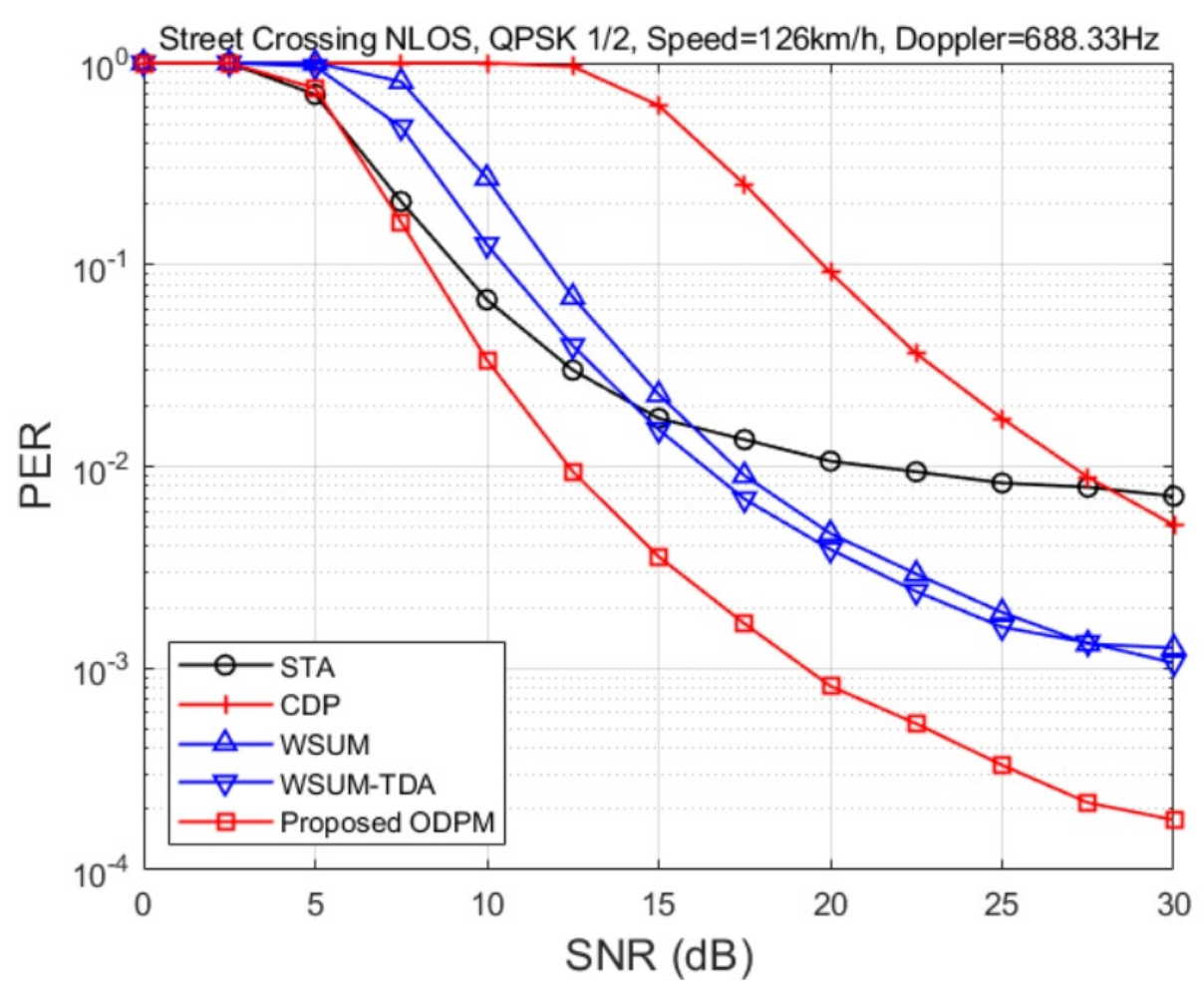

Fig. 2. Comparison of PER performance of the proposed ODPM with conventional CE schemes in Street Crossing NLOS channel model with a relative speed of 126km/h (QPSK 1/2, R=2).

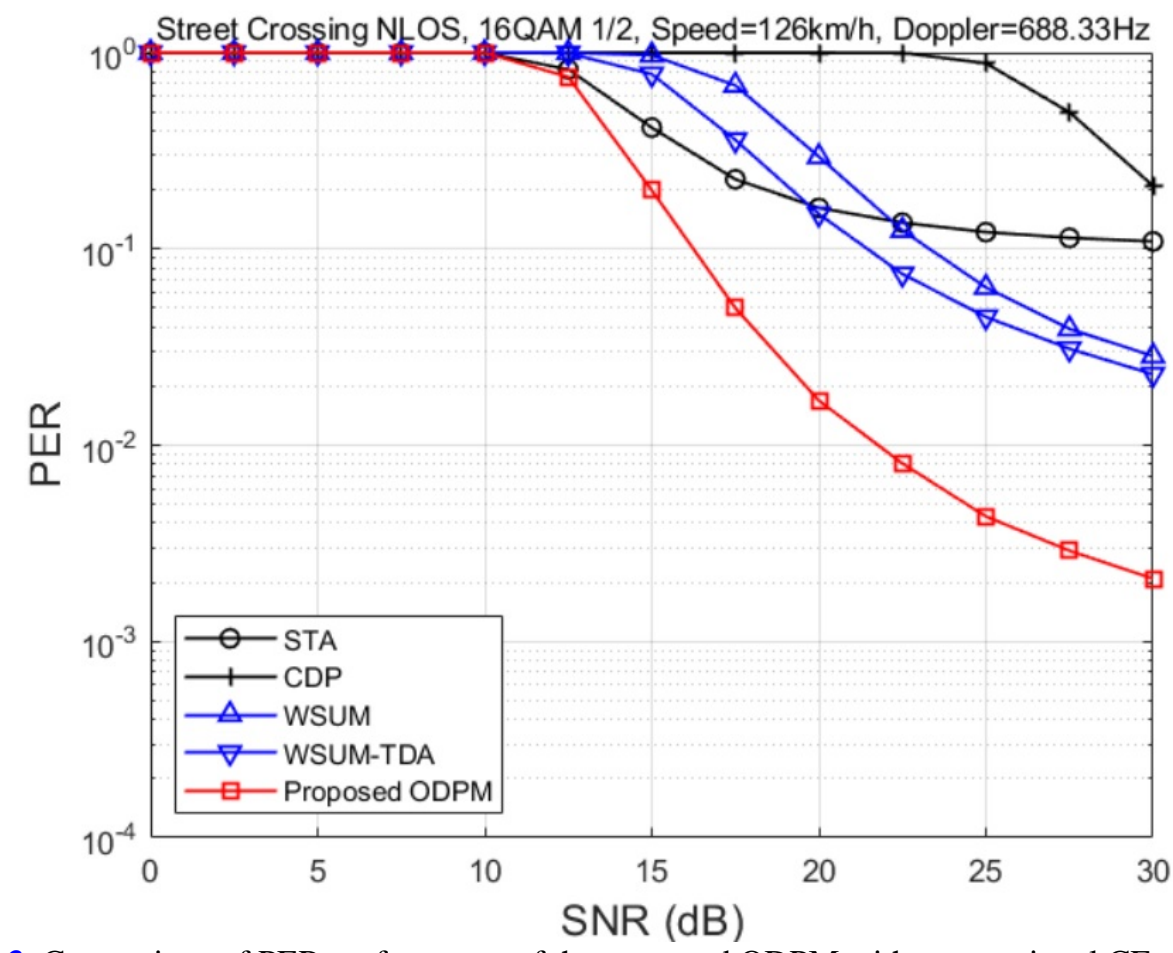

Fig. 3. Comparison of PER performance of the proposed ODPM with conventional CE schemes in Street Crossing NLOS channel model with a relative speed of 126km/h (16QAM 1/2, R=2). 


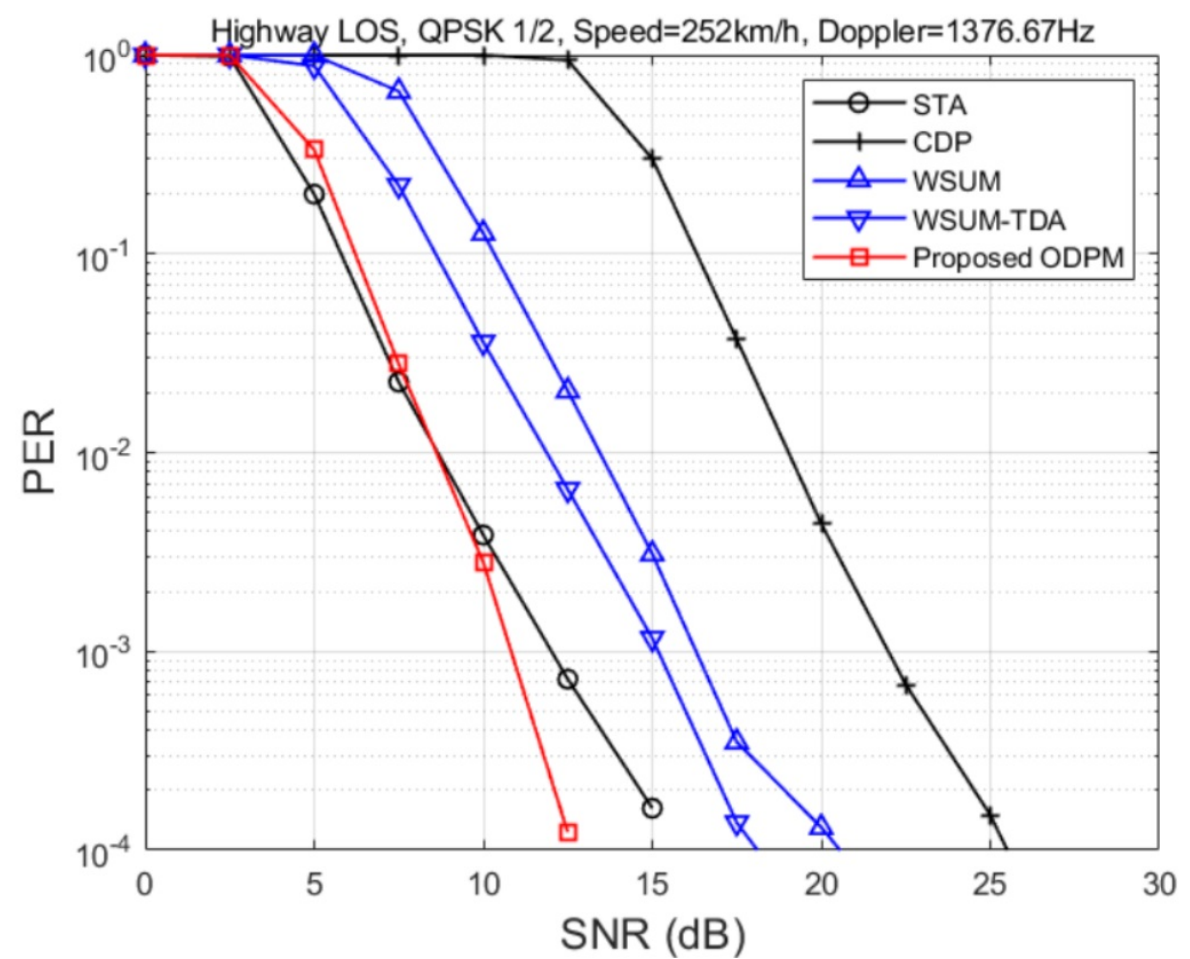

Fig. 4. Comparison of PER performance of the proposed ODPM with conventional CE schemes in Highway LOS channel model with a relative speed of 252km/h (QPSK 1/2, R=2).

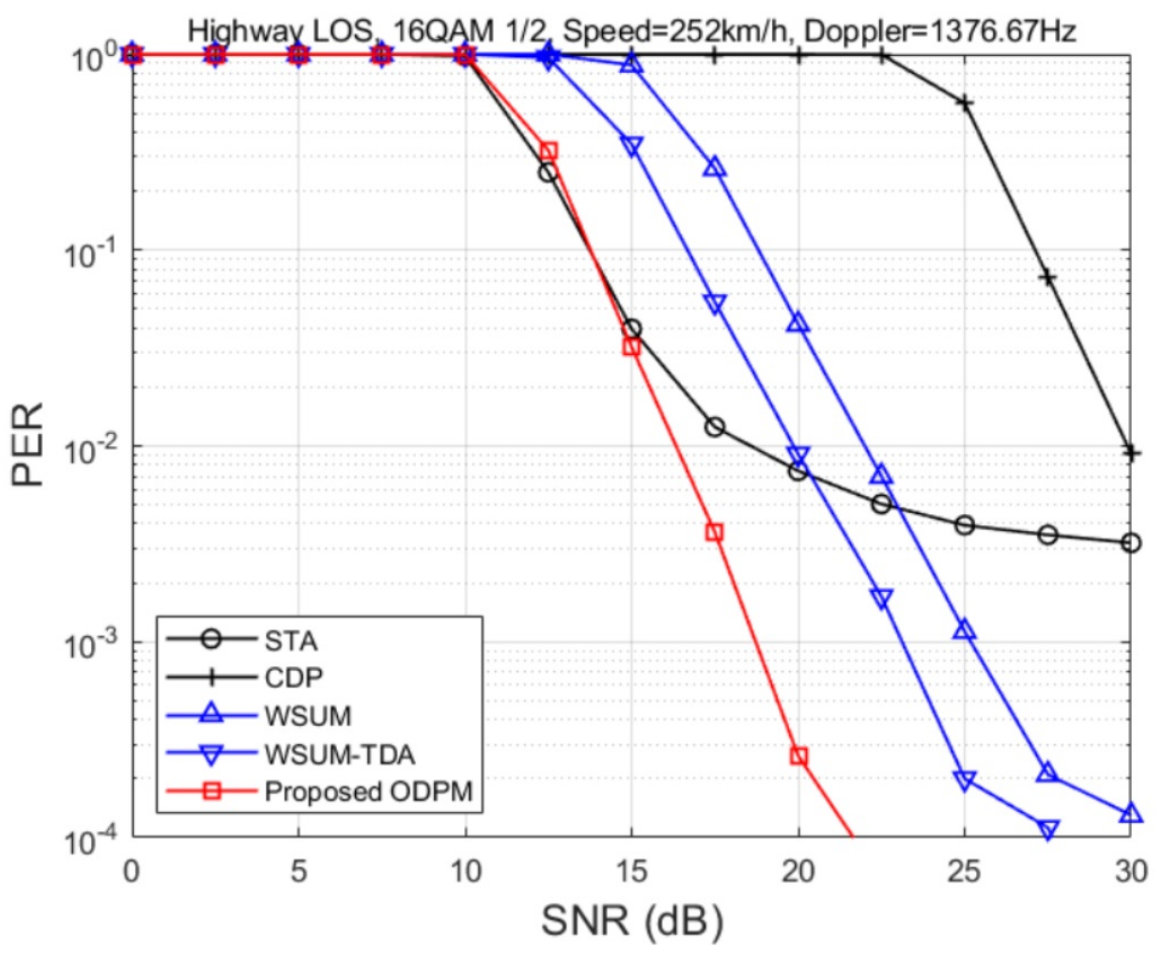

Fig. 5. Comparison of PER performance of the proposed ODPM with conventional CE schemes in Highway LOS channel model with a relative speed of 252km/h (16QAM 1/2, R=2). 


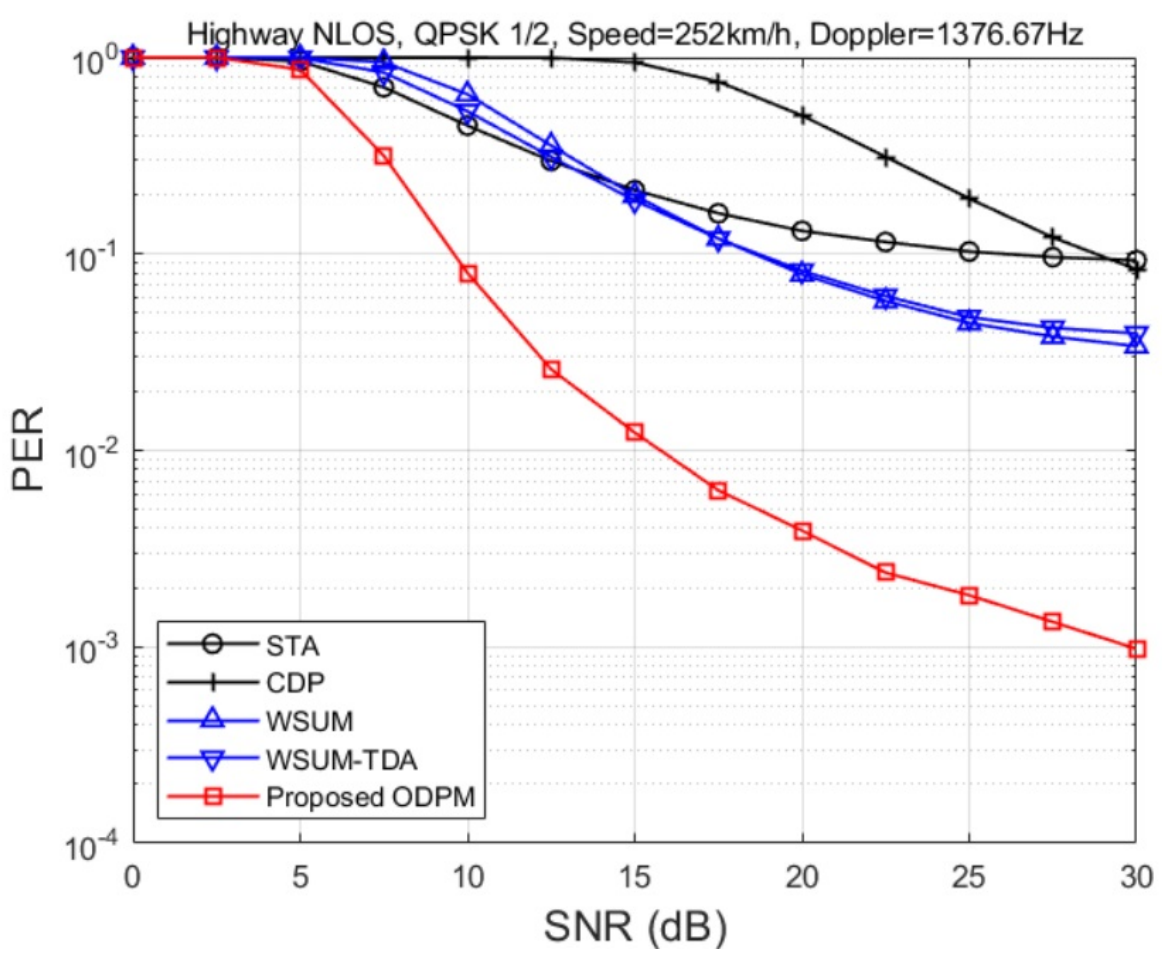

Fig. 6. Comparison of PER performance of the proposed ODPM with conventional CE schemes in Highway NLOS channel model with a relative speed of $252 \mathrm{~km} / \mathrm{h}(\mathrm{QPSK} 1 / 2, \mathrm{R}=2)$.

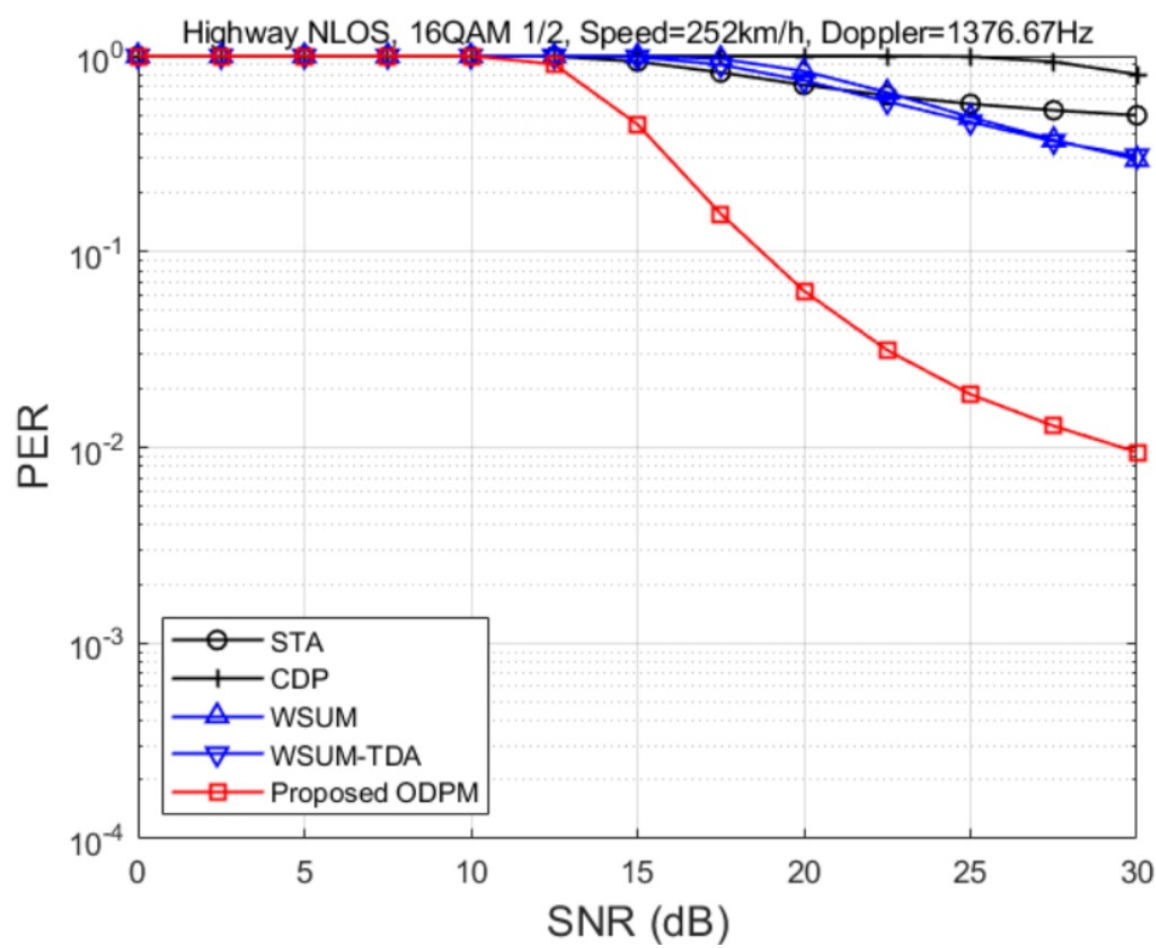

Fig. 7. Comparison of PER performance of the proposed ODPM with conventional CE schemes in Highway NLOS channel model with a relative speed of 252km/h (16QAM 1/2, R=2). 


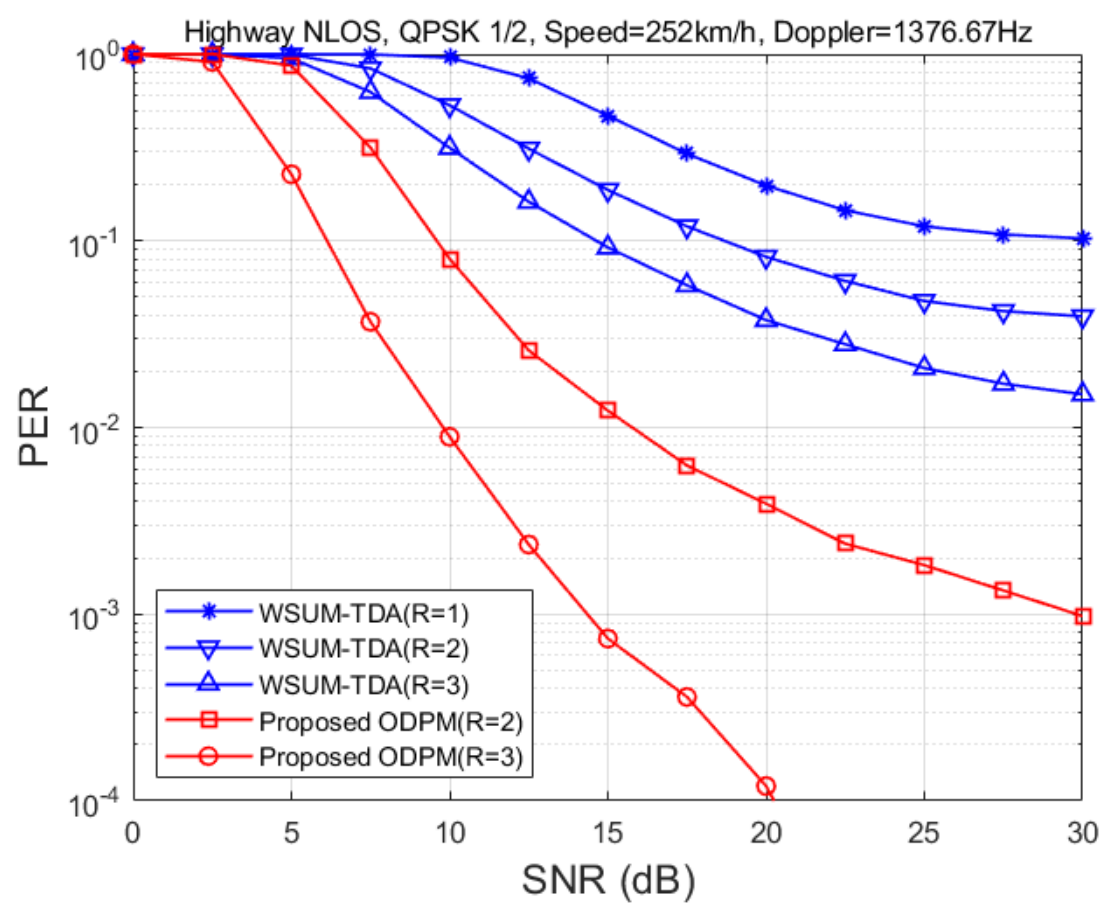

Fig. 8. Comparison of PER performance of the proposed ODPM with WSUM-TDA as the number of antennas varies in Highway NLOS channel model with a relative speed of $252 \mathrm{~km} / \mathrm{h}$ (QPSK 1/2).

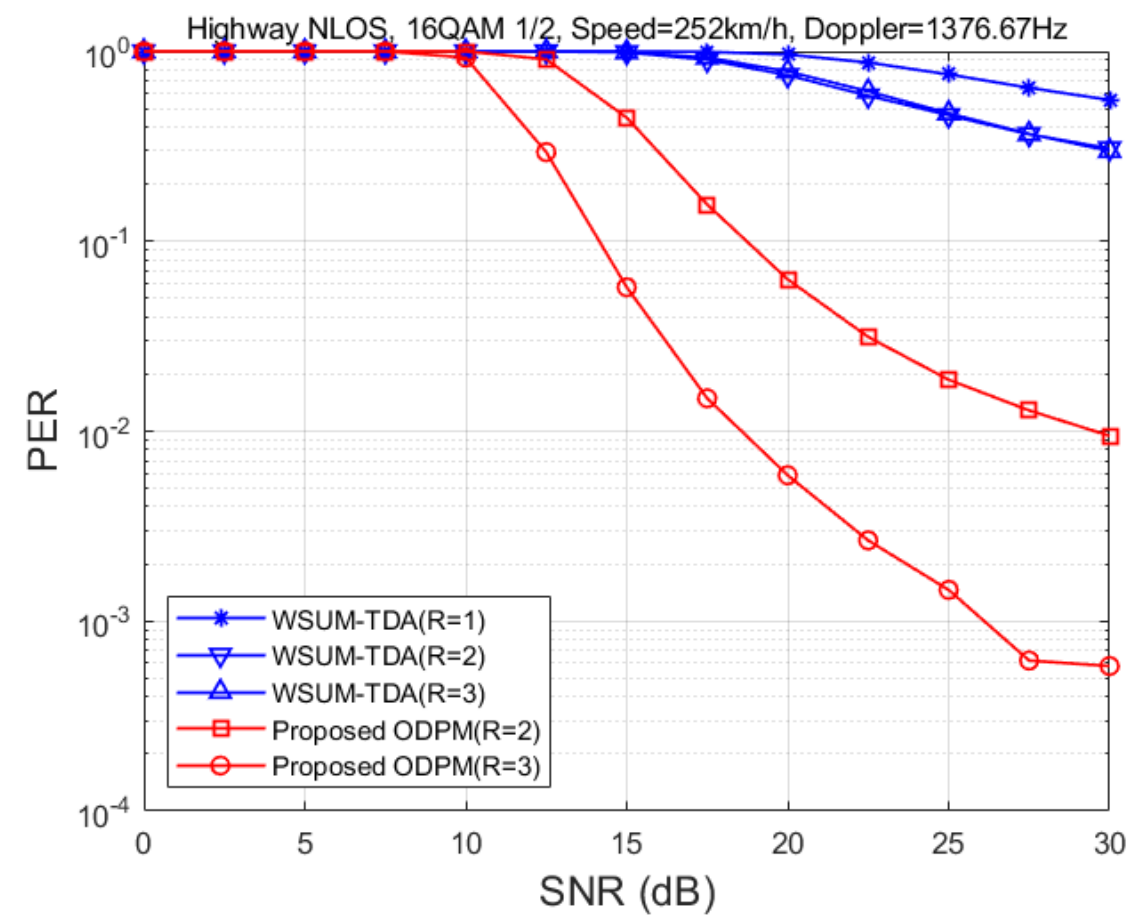

Fig. 9. Comparison of PER performance of the proposed ODPM with WSUM-TDA as the number of antennas varies in Highway NLOS channel model with a relative speed of 252km/h (16QAM 1/2). 


\section{Conclusions}

In this paper, as a novel CE technique suitable for WAVE systems based on IEEE 802.11p, ODPM for multiple receiving antennas is proposed and its PER performance is demonstrated through numerical simulations. ODPM is superior to various conventional CE methods with regard to the PER performance. Especially, its euperiority is verified at all SNR regions, regardless of the modulation type.

\section{Acknowledgements}

This work was supported by the National Research Foundation of Korea (NRF) grant funded by the Korea government (MSIT) (No. 2020R1A2C1005260, NRF-2021R1A2C2012558).

\section{References}

[1] IEEE guide for wireless access in vehicular environments(WAVE) architecture, IEEE Standard 1609.0-2013, pp. 1-78, Mar. 2014.

[2] Daein Jeong, "Priority Broadcast Modeling of IEEE 802.11p MAC with Channel Switching Operation,” IEICE Trans. Commun., vol.E102-B, no.9, pp.1895-1903, Sept. 2019.

Article (CrossRef Link)

[3] Seunghwan Baek, Inkyu Lee, and Changick Song, "A New Data Pilot-Aided Channel Estimation Scheme for Fast Time-Varying Channels in IEEE 802.11p Systems,” IEEE Trans. Veh. Technol., vol. 68, no. 5, pp. 5169-5172, MAY 2019. Article (CrossRef Link)

[4] J. Zhao, X. Cheng, M. Wen, B. Jiao, and C. X. Wang, "Channel Estimation Schemes for IEEE 802.11p Standard,” IEEE Intell. Transp. Syst. Mag., vol.5, no.4, pp.38-49, winter 2013. Article (CrossRef Link)

[5] Yoon Kyeong Kim, Jang-Mi Oh, Yoo Ho Shin, Cheol Mun, "Time and frequency domain channel estimation scheme for IEEE 802.11p," in Proc. of IEEE 17th International Conference on Intelligent Transportation Systems (ITSC 2014), Qingdao, China, pp.1085-1090, 8-11 Oct. 2014. Article (CrossRef Link)

[6] Cheol Mun, Jooyoung Choi, Yeona Kim, Min Baek, Gilwon Seo, and Kyunbyoung Ko, "Cell Planning and Deployment for IEEE 802.11p/WAVE Network,” IEEE Intell. Transp. Syst. Mag., vol.7, no.4, pp.49-57, winter 2015. Article (CrossRef Link)

[7] Joo-Young Choi, Cheol Mun, and Jong-Gwan Yook, "Adaptive Channel Estimation Based on a Decision Method Using a Long Preamble for the IEEE 802.11p," in Proc. of 2017 IEEE 85th Vehicular Technology Conference (VTC2017-Spring), Sydney, Australia, 4-7 Jun. 2017.

Article (CrossRef Link)

[8] Cho-Eun Park and Kyun-Byoung Ko, "Advanced Channel Estimation Schemes Using CDP based Updated Matrix for IEEE802.11p/WAVE Systems," International Journal of Contents, vol.14, no.1, pp.39-44, Mar. 2018. Article (CrossRef Link)

[9] DongSeon Jang and Kyunbyoung Ko, "Advanced Channel Estimation Method for IEEE 802.11p/WAVE System,” International Journal of Contents, vol.15, no.4, pp.27-35, Dec. 2019. Article (CrossRef Link)

[10] G. Maier, A. Paier, and C. F. Mecklenbrauker, "Performance evaluation of IEEE 802.11p infrastructure-to-vehicle real-world measurements with receive diversity," in Proc. of 2012 8th International Wireless Communications and Mobile Computing Conference (IWCMC). Limassol, Cyprus, pp.1113-1118, 27-31 Aug. 2012. Article (CrossRef Link)

[11] S. Han, K. Zhao, L.-Q. Yang, and X. Cheng, "Performance evaluation for multi-antenna vehicular communication based on IEEE 802.11p standard," in Proc. of 2016 International Conference on Computing, Networking and Communications (ICNC), Kauai, USA, pp.1-5, 15-18 Feb. 2016. Article (CrossRef Link) 
[12] R. Budde, S. Nowak, and R. Kays, "Study of Receiver Concepts for Vehicular Channels in IEEE 802.11p Systems," in Proc. of 2013 IEEE 77th Vehicular Technology Conference (VTC2013Spring), Dresden, Germany, pp.1-5, 2-5 Jun. 2013. Article (CrossRef Link)

[13] Gihoon Ryu and Kyunbyoung Ko, "MRC based Channel Estimation method using Data Pilot Construction and Reliability Test in IEEE 802.11p/WAVE systems with Multiple Receive Antennas," in Proc. of 2018 International Conference on Convergence Content (ICCC 2018), Jeju, Korea, Dec. 17-19, 2018.

[14] Junseop Kim and Sungmook Lim, "Iterative Channel Estimation Scheme based on MRC Packing Channel Estimation Scheme in Multiple Receive Antennas Environment for PER Enhancement of IEEE 802.11p / WAVE System," in Proc. of 2018 International Conference on Convergence Content (ICCC 2018), Jeju, Korea, Dec. 17-19, 2018.

[15] Malik Kahn, "IEEE 802.11 Regulatory SC DSRC Coexistence Tiger Team - V2V Radio Channel Models,” [PowerPoint Slides]. Doc. IEEE 802.11-14, Feb. 2014.

[16] Yu-Kuan Chang, Fang-Biau Ueng, Ye-Shun Shen and Chih-Yuan Liao, “ Joint Kalman Channel Estimation and Turbo Equalization for MIMO OFDM Systems over Fast Fading Channels," KSII Transactions on Internet and Information Systems, vol. 13, no. 11, pp. 5394-5409, Nov. 2019. Article (CrossRef Link)

[17] Ahmed Ali, Wang Dong, Li Renfa and Esraa Eldesouky, "ISI and PAPR Immune IEEE 802.11p Channels Based on Single-Carrier Frequency Domain Equalizer, ” KSII Transactions on Internet and Information Systems, vol. 10, no. 11, pp.5513-5529, Nov. 2016. Article (CrossRef Link)

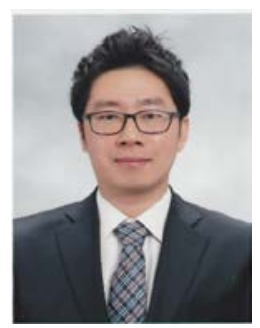

Sungmook Lim received the B.S. and Ph.D. degrees in Electrical and Electronic Engineering at Yonsei University, Seoul, Korea in 2005 and 2012, respectively. From September 2012 to March 2014, he was a Postdoctoral Fellow at Yonsei University where his research interests were in 5G wireless communications. Since March 2014, he joined at Korea National University of Transportation. Currently, he is an Assistant Professor at the Department of electronics Engineering and the Department of IT-Energy Convergence (BK21 FOUR). His current research interests include the field of 5G wireless communications focusing on multicarrier and multi-antenna systems, cooperative relaying, and ITS.

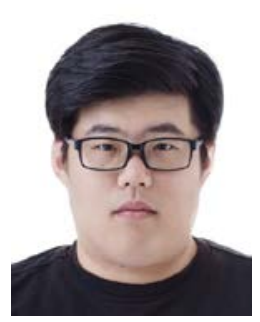

Gihoon Ryu received the B.S. degree in Department of Control and Instrumentation Engineering at Korea National University of Transportation in 2017 and the M.S. degree in Department of IT Convergence at Korea National University of Transportation in 2019. His current research interests include wireless communications, cooperative relaying, and C-ITS.

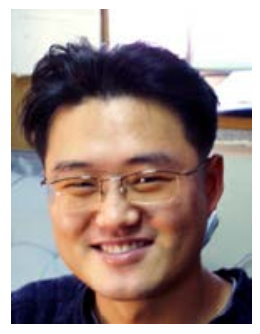

Kyunbyoung Ko(corresponding author) received the B.S., M.S., and Ph.D. degrees in Electrical and Electronic Engineering at Yonsei University, Seoul, Korea in 1997, 1999, and 2004, respectively. From March 2004 to February 2007, he was a senior engineer in Samsung Electronics, Suwon, Korea where he developed Mobile WiMAX systems for broadband wireless services. Since March 2007, he joined at Korea National University of Transportation. Currently, he is a Professor at the Department of electronics Engineering and the Department of IT-Energy Convergence (BK21 FOUR). His current research interests include the field of wireless communications focusing on multicarrier and multi-antenna systems, cooperative relaying, and C-ITS. He is a member of IEIE, KICS, KOCON, IEEE, and IEICE. 